\title{
New Exact Solutions for a Class of High-order Dispersive Cubic-quintic Nonlinear Schrödinger Equation
}

\author{
Ying Huang ${ }^{1} \&$ Peng Liu ${ }^{1}$ \\ ${ }^{1}$ School of Mathematics and Statistics, Chuxiong Normal University, Chuxiong, P.R.China \\ Correspondence: Ying Huang, School of Mathematics and Statistics, Chuxiong Normal University, Chuxiong, \\ P.R.China. E-mail: huang11261001@163.com
}

Received: September 27, 2014 Accepted: October 11, 2014 Online Published: November 6, 2014

doi:10.5539/jmr.v6n4p104 URL: http://dx.doi.org/10.5539/jmr.v6n4p104

\begin{abstract}
In the paper, the exact solutions to the cubic-quintic nonlinear Schrödinger equation with third and fourth-order dispersion terms is considered. The improved homogeneous balance method is used for constructing a series of new exact envelop wave solutions, including envelop solitary wave solutions, envelop periodic wave solutions and an envelop rational solution.
\end{abstract}

Keywords: exact solution, high dispersive, nonlinear Schrödinger equation

\section{Introduction}

Since third and fourth-order dispersion terms play a crucially important role in describing the propagation of extremely short pulses, the generalized nonlinear Schrödinger equation

$$
i q_{z}-\frac{\beta_{2}}{2} q_{t t}+\gamma_{1}|q|^{2} q=i \frac{\beta_{3}}{6} q_{t t t}+\frac{\beta_{4}}{24} q_{t t t t}-\gamma_{2}|q|^{4} q
$$

is taken as a model for a sub-picoseconds pulse propagation in a medium which exhibits a parabolic nonlinearity law (Shundong, 2007, Karpman,1997). In particular, $q(z, t)$ is the slowly varying envelope of the electromagnetic field, $\beta_{2}$ is the parameter of the group velocity dispersion, $\beta_{3}$ and $\beta_{4}$ are third-order and fourth-order dispersion, respectively, $\gamma_{1}$ and $\gamma_{2}$ are the nonlinearity coefficients (Karpman, 1998). As to this equation, its modulation instability of optical wave was numerically investigated (Woo-Pyo,2002), the relative exact analytic solutions were studied very few, the researchers mainly studied its several special cases because of itself complications. For the case $\beta_{3}=0$, a series of analytical exact solutions are constructed by means of F-expansion method (Gui-Qiong, 2011). In the case $\beta_{3}=\gamma_{1}=0$, a dark solitary wave solution with amplitude only depending on the time was presented (Azzouzi,2014). In contrast, when $\beta_{3}=\beta_{4}=\gamma_{2}=0$, various types exact solution were discovered (Azzouzi, 2009, Huiqing,2009, Wenxiu,2009, Anjan,2010).

Two exact periodic solutions and two kink soliton solutions had been constructed with the homogeneous balance method (Shundong, 2007). Here, we construct new the exact solutions with the aid of the improved homogeneous balance method. In section 2, we introduce the general method for solving complex partial differential equations, and solve the new analytical solutions to high-order dispersive cubic-quintic nonlinear Schrödinger equation in section 3 .

\section{The Main Method}

In this section, we propose a general method to obtain exact solutions for complex nonlinear partial differential equations, based on the homogeneous balance method.

Consider a given complex partial differential equation with independent variables $z$ and $t$

$$
H\left(q, q_{z}, q_{z z}, \ldots q_{t}, \ldots\right)=0
$$

where $H$ is in general a complex polynomial in $q$ and its various partial derivatives. The main steps are:

First. Taking the transformation 


$$
q(x, t)=u(\xi) e^{i\left(p_{1} z+p_{2} t+c_{0}\right)}, \xi=k_{1} z+k_{2} t+\xi_{0},
$$

where $k_{i}, p_{i}, i=1,2$ are constants to be determined, $c_{0}, \xi_{0}$ are arbitrary constants. Substituting (3) into (2) yields a complex ordinary differential equation, in which the real and imaginary parts read, respectively,

$$
\begin{array}{r}
H_{R}\left(u, u^{\prime}, u^{\prime \prime}, \ldots\right)=0, \\
H_{I}\left(u, u^{\prime}, u^{\prime \prime}, \ldots\right)=0 .
\end{array}
$$

Second. The next crucial step is to take

$$
u(\xi)=\sum_{i=1}^{n} a_{i} F^{i}(\xi)
$$

where $a_{i}$ are constants to be determined later, $n$ is a positive integer that can be determined by balancing the highest-order derivative terms with the highest power nonlinear terms in the equations, and $F(\xi)$ satisfies the first kind elliptic equation

$$
\left(F^{\prime}(\xi)\right)^{2}=b_{1}+b_{2} F^{2}(\xi)+b_{3} F^{4}(\xi)
$$

where $b_{i}$ are arbitrary constants, but $b_{3}$ is not equal to zero.

Third. Substitute Eq.(7) with (6) into (4) and (5), the left hand sides of (4) and (5) can be converted into two finite series in $F^{i}$, respectively, and equating each coefficient of $F^{i}$ to zero yields a system of algebraic equations for $k_{i}, p_{i}, a_{i}$. Solve it, $k_{i}, p_{i}$ and $a_{i}$ can be expressed by $b_{i}$ and the coefficients of Eq.(2).

Fourth. Solving Eq.(7) and compounding it's solutions with (6) and (3), we can obtain a series of envelop solitary wave solutions, triangle functions and rational solutions of Eq.(2).

\section{Exact solutions to Eq. (1)}

In this section, we will construct the exact solutions for Eq.(1).

Making the transformation

$$
q=u(\xi) e^{i(\omega t+\theta z)}, \xi=\mu t+\alpha z+\xi_{0},
$$

for Eq.(1), where $\omega, \theta, \mu$ and $\alpha$ are constants to be determined. Then separating the real and imaginary parts of the resulting complex ordinary differential equation, we obtain

$$
\begin{gathered}
\frac{\beta_{4} \mu^{4}}{24} u_{\xi \xi \xi \xi}+\mu^{2}\left(\frac{\beta_{2}}{2}-\frac{\beta_{4}}{4} \omega^{2}-\frac{\beta_{3}}{2} \omega\right) u_{\xi \xi}+\left(\frac{\beta_{4}}{24} \omega^{4}+\frac{\beta_{3}}{6} \omega^{3}+\theta-\frac{\beta_{2}}{2} \omega^{2}\right) u-\gamma_{1} u^{3}-\gamma_{2} u^{5}=0 ; \\
\mu^{3}\left(\frac{\beta_{3}}{6}+\frac{\beta_{4}}{6} \omega\right) u_{\xi \xi \xi}+\left(\beta_{2} \omega \mu-\alpha-\frac{\beta_{3}}{2} \mu \omega^{2}-\frac{\beta_{4}}{6} \mu \omega^{3}\right) u_{\xi}=0 .
\end{gathered}
$$

Especially, the method of dealing with the above relations differs from Shundong's (Shundong,2007). Setting

$$
\left\{\begin{array}{l}
\mu^{3}\left(\frac{\beta_{3}}{6}+\frac{\beta_{4}}{6} \omega\right)=0, \\
\beta_{2} \omega \mu-\alpha-\frac{\beta_{3}}{2} \mu \omega^{2}-\frac{\beta_{4}}{6} \mu \omega^{3}=0 .
\end{array}\right.
$$

we finally get

$$
\beta_{4} \mu^{4} u_{\xi \xi \xi \xi}+6 \mu^{2}\left(2 \beta_{2}-\beta_{4} \omega^{2}-2 \beta_{3} \omega\right) u_{\xi \xi}+\left(\beta_{4} \omega^{4}+4 \beta_{3} \omega^{3}+24 \theta-12 \beta_{2} \omega^{2}\right) u-24 \gamma_{1} u^{3}-24 \gamma_{2} u^{5}=0 .
$$

Next, our task is to solve the above ordinary differential equation, by balancing the highest order derivative terms and the highest power nonlinear terms, we choose the ansatz

$$
u=a F(\xi), a \neq 0,
$$


where $a$ is a constant to be determined, and $F(\xi)$ must satisfy the following auxiliary equation

$$
\left(\frac{d F}{d \xi}\right)^{2}=b+c F^{2}+d F^{4},
$$

where $b, c$ and $d$ are arbitrary constants. Substituting (12) with (13) into (11) and letting each coefficient of $F^{i}(i=1,2,3)$ to be zero, we get algebraic equations

$$
\left\{\begin{array}{l}
\beta_{4} \mu^{4}\left(c^{2}+12 b d\right)+6 \mu^{2}\left(2 \beta_{2}-\beta_{4} \omega^{2}-2 \beta_{3} \omega\right) c+\beta_{4} \omega^{4}+4 \beta_{3} \omega^{3}+24 \theta-12 \beta_{2} \omega^{2}=0 \\
20 c d \beta_{4} \mu^{4}+12 d \mu^{2}\left(2 \beta_{2}-\beta_{4} \omega^{2}-2 \beta_{3} \omega\right)-24 \gamma_{1} a^{2}=0 \\
24 d^{2} \beta_{4} \mu^{4}-24 \gamma_{2} a^{4}=0
\end{array}\right.
$$

Solving the algebraic equations (10) and (14) yields

$$
\begin{gathered}
\omega=-\frac{\beta_{3}}{\beta_{4}} ; \quad a= \pm \frac{1}{\beta_{4}} \sqrt{\frac{6 \gamma_{1} d \beta_{4}^{2}-3 \gamma_{2}|d| \sqrt{\frac{\beta_{4}}{\gamma_{2}}}\left(2 \beta_{2} \beta_{4}+\beta_{3}^{2}\right)}{5 c \gamma_{2}}} \\
\mu= \pm \frac{1}{\beta_{4}} \sqrt{\frac{6 \gamma_{1}|d| \beta_{4} \sqrt{\frac{\beta_{4}}{\gamma_{2}}}-3 d\left(2 \beta_{2} \beta_{4}+\beta_{3}^{2}\right)}{5 c d}} ; \alpha=-\mu \frac{\beta_{3}\left(3 \beta_{2} \beta_{4}+\beta_{3}^{2}\right)}{3 \beta_{4}^{3}} \\
\theta=\frac{\beta_{3}^{2}\left(\beta_{3}^{2}+4 \beta_{2} \beta_{4}\right)}{8 \beta_{4}^{3}}-\frac{\beta_{4}\left(c^{2}+12 b d\right)}{24} \mu^{4}-\frac{c\left(2 \beta_{2} \beta_{4}+\beta_{3}^{2}\right)}{4 \beta_{4}} \mu^{2} .
\end{gathered}
$$

Solving $F(\xi)$ from (13) one by one and substituting them into (15) together with (8) yields the following exact solutions.

Envelop period wave solutions of Eq.(1) have the form:

$$
\begin{aligned}
& q=a \sqrt[4]{\frac{b}{d}} \tan \sqrt[4]{b d} \xi e^{i(\omega t+\theta z)}, \\
& q=a \sqrt[4]{\frac{b}{d}} \cot \sqrt[4]{b d} \xi e^{i(\omega t+\theta z)},
\end{aligned}
$$

where $c=2 \sqrt{b d}$.

Envelop kink soliton solutions are given by:

$$
\begin{aligned}
& q=a \sqrt[4]{\frac{b}{d}} \tanh \sqrt[4]{b d} \xi e^{i(\omega t+\theta z)} \\
& q=a \sqrt[4]{\frac{b}{d}} \operatorname{coth} \sqrt[4]{b d} \xi e^{i(\omega t+\theta z)}
\end{aligned}
$$

where $c=-2 \sqrt{b d}$.

Envelop periodic wave solutions of the form:

$$
\begin{aligned}
& q=a \sqrt{\frac{-c}{d}} \sec \sqrt{-c} \xi e^{i(\omega t+\theta z)}, \\
& q=a \sqrt{\frac{-c}{d}} \csc \sqrt{-c} \xi e^{i(\omega t+\theta z)},
\end{aligned}
$$

where $b=0, c<0, d>0$. 
Envelop bell soliton solutions are given by:

$$
q=a \sqrt{\frac{-c}{d}} \operatorname{sech} \sqrt{c} \xi e^{i(\omega t+\theta z)},
$$

where $b=0, c>0, d<0$,

and also

$$
q= \pm \sqrt{\frac{c}{d}} \operatorname{csch} \sqrt{c} \xi e^{i(\omega t+\theta z)}
$$

where $b=0, c>0, d>0$.

Envelop rational solution is

$$
q=a \frac{ \pm 1}{\sqrt{d} \xi} e^{i(\omega t+\theta z)},
$$

where $b=c=0, d>0$, and this is singular at $\xi=0$.

In (16)-(24), $\xi=\mu t+\alpha z+\xi_{0}, a, \theta, \omega, \alpha$ and $\mu$ are given by (15).

\section{Conclusions}

In summary, Shundong, Z. early considered the exact envelop wave solutions to the high dispersive cubic-quintic nonlinear Schrödinger equation which describes the propagation of extremely short pulses. Based on the improved homogeneous balance method, we obtain some exact envelop wave solutions by taking the first elliptic equation as auxiliary ordinary differential equation, some physical phenomena of extremely short pulses can be better understood with the help of these new solutions.

\section{Acknowledgements}

This work was supported by Chinese Natural Science Foundation Grant (11261001) and Yunnan Provincial Department of Education Research Foundation Grant (2012Y130).

\section{References}

Anjan, B., Allison, M., Daniela, M., Fayequa, M., \& Keka, C. B. (2010). An exact solution for the modified nonlinear Schrödingers equation for Davydov solitons in -helix proteins. Mathematical Biosciences, 227(1), 68-71. http://dx.doi.org/10.1016/j.mbs.2010.05.008

Azzouzi, F., Triki, H., \& Grelu, P. (2014). Dipole soliton solution for the homogeneous high-order nonlinear Schrödinger equation with cubicCquinticCseptic non-Kerr terms. Applied Mathematical Modelling, (in press) http://dx.doi.org/10.1016/j.apm.2014.08.011

Azzouzi, F., Triki, H., Mezghich, K., \& EI Akrmi, A. (2009). Solitary wave solutions for higher dispersive cubicquintic nonlinear Schrödinger equation. Chaos Solitons Fract., 39(3), 1304-1307. http://dx.doi.org/10.1016/j.chaos.2007.06.024

Gui-Qiong, X. (2011). New types of exact solutions for the fourth-order dispersive cubicCquintic nonlinear Schrödinger equation. Applied Mathematics and Computation, 217(12), 5967-5971. http://dx.doi.org/10.1016/j.amc.2010.12.008

Huiqun, Z. (2009). New exact complex travelling wave solutions to nonlinear Schrödinger(NLS) equation. Commun Nonlinear Sci Numer Simulat., 14(3), 668-673. http://dx.doi.org/10.1016/j.cnsns.2007.11.014

Karpman, V. I., \& Shagalov, A. G. (1997). Solitons and their stability in high dispersive system.I. Fourthorder nonlinear Schrödinger-type equations with power-law nonlinearities. Physics letter A, 228(1-2), 59-65. http://dx.doi.org/10.1016/S0375-9601(97)00063-7

Karpman, V. I. (1998). Evolution of solitons described by high-order nonlinear Schrödinger equations. Physics letter A, 244(5), 397-400. http://dx.doi.org/10.1016/S0375-9601(98)00251-5

Shundong, Z. (2007). Exact solutions for the high-order dispersive cubic-quintic nonlinear Schrödinger equation by the extended hyperbolic auxiliary equation method. Chaos Solitons Fract, 34(5), 1608-1612.

http://dx.doi.org/10.1016/j.chaos.2006.05.001 
Wenxiu, M., \& Min, C. (2009). Direct search for exact solutions to the nonlinear Schrödinger equation. Applied Mathematics and Computation, 215(8), 2835-2842. http://dx.doi.org/10.1016/j.amc.2009.09.024

Woo-Pyo, H. (2002). Modulational instability of optical waves in the high dispersive cubic-quintic nonlinear Schrödinger equation. Opt Commum., 213(1-3), 173-182. http://dx.doi.org/10.1016/S0030-4018(02)02046-1

\section{Copyrights}

Copyright for this article is retained by the author(s), with first publication rights granted to the journal.

This is an open-access article distributed under the terms and conditions of the Creative Commons Attribution license (http://creativecommons.org/licenses/by/3.0/). 